\title{
De novo Growth of Pre-peritoneal Leiomyoma of the Abdominal Wall: A Diagnostic Challenge
}

\author{
Dr Dinesh M Gunasagar ${ }^{1}$, Dr Ashutosh Sahewalla ${ }^{2}$, Dr Chatura K R $\mathbf{R}^{3}$ \\ ${ }^{1}$ Professor, Department of Surgery, JJM Medical College, Davangere \\ ${ }^{2}$ Post graduate, Department of Surgery, JJM Medical College, Davangere \\ ${ }^{3}$ Professor, Department of Pathology, JJM Medical College, Davangere
}

\begin{abstract}
Extrauterine or extraintestinal leiomyomas are extremely uncommon especially in the pre-peritoneal area or within the anterior abdominal wall muscles. There is paucity of reports of isolated de novo pre-peritoneal wall leiomyomas in the literature. We therefore report one such case of pre-peritoneal abdominal wall leiomyoma occurring in post menopausal woman aged 55 years with no history of prior abdominal operations.
\end{abstract}

Keywords: Abdominal wall tumour, Pre-peritoneal leiomyoma

\section{Introduction}

Fibroid or the leiomyoma is the commonest benign tumour of the female reproductive tract and is seen in $20 \%$ of women of reproductive age. Uterus is the most common site followed by broad ligament, ovaries, and vagina and very rarely in the anterior abdominal wall. Abdominal wall leiomyomata are uncommon and are thought to follow seeding after resection of uterine fibroids. Here we present a rare case of isolated leiomyoma of the anterior abdominal wall in pre-peritoneal space in a woman with no previous history of any gynaecological surgeries.

\section{Case Report}

A 55 year old multiparous $\left(\mathrm{G}_{4} \mathrm{P}_{4}\right)$, postmenopausal lady presented with pain abdomen for the past 6 months which was dull aching type more in the lower abdomen. There was no history of any previous surgery or gynaecologic interventions. She was not on hormone replacement therapy.

On examination, there was a smooth globular, non-tender, soft mass present in the hypogastric region measuring about $10 * 8 \mathrm{~cm}$. Inferior margin was not well made out. Clinically it was an intra-abdominal mass with minimal mobility. Pelvic examination showed mass not arising from uterus or ovary.

Her haematological and routine biochemistry investigations were within normal limits. CA-125 was found to be normal. Ultrasonography of abdomen and pelvis (Fig.1) showed a large oblong solid mass $12.4 * 8.7 \mathrm{cms}$ in pelvic cavity predominantly to left suggestive of ?malignant ovarian mass/ sarcoma.

Contrast enhanced CT scan of abdomen and pelvis (Fig.2) revealed a well defined lower abdominal solid mass in the midline extending on both sides. The mass was measuring $19.8 * 9.8 \mathrm{cms}$. The mass was seen antero-superior to bladder, displacing the adjacent bowel. The lesion was seen separately from bladder/ bowel/ uterus/ovary suggesting the possibility of desmoid/ GIST/ lymphoma.

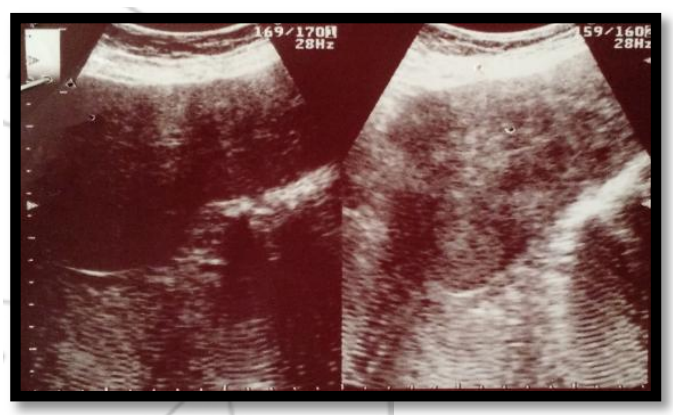

Figure 1: Ultrasound showing solid lesion in the pelvis close to left ovary.

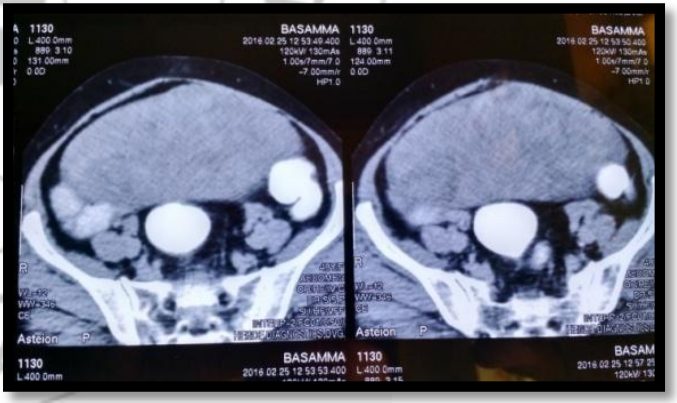

Figure 2: CT scan showing solid mass lesion in the midline extending on either sides.

USG guided Fine Needle Aspiration Cytology showed occasional spindle cells and vacuolated polygonal cells. Malignant cells were not seen. Possibility of desmoid tumour was considered at that stage.

Patient underwent exploratory laparotomy under general anaesthesia. Abdomen was opened with a lower midline incision. After opening the skin and subcutaneous tissues, recti were separated entering the pre-peritoneal space revealing a well-encapsulated mass (Fig.3). The mass was dissected completely from the surrounding structures. After the mass was completely excised (Fig.4), the peritoneum was deliberately opened for inspection of intra-abdominal organs which were found to be normal. Primary anatomical closure of the incision was done. 


\section{International Journal of Science and Research (IJSR) \\ ISSN (Online): 2319-7064}

Index Copernicus Value (2015): 78.96 | Impact Factor (2015): 6.391

The mass was measuring $18 \times 10 \times 5 \mathrm{~cm}$ (Fig.5), weighing $1.5 \mathrm{kgs}$ which was soft in consistency with prominent overlying blood vessels. Patient made uneventful recovery and was discharged after 10days. After 8months of follow up patient was doing well without any evidence of recurrence.

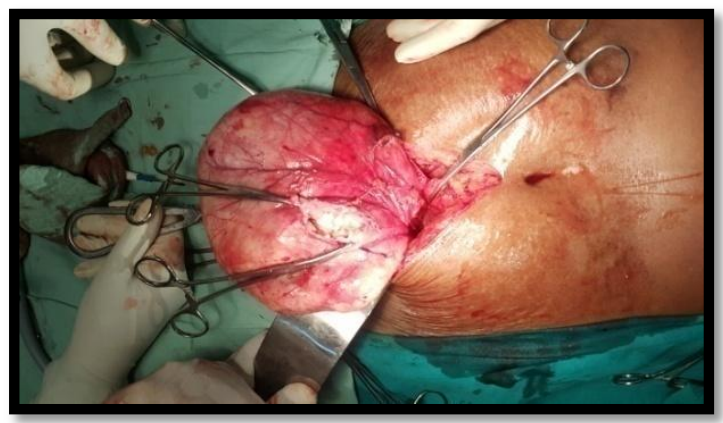

Figure 3: Mass being freed from peritoneum

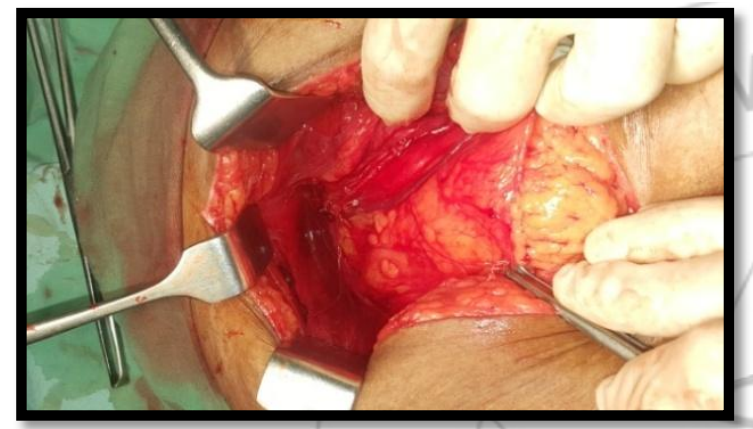

Figure 4: Pre-peritoneal space after removal of the mass

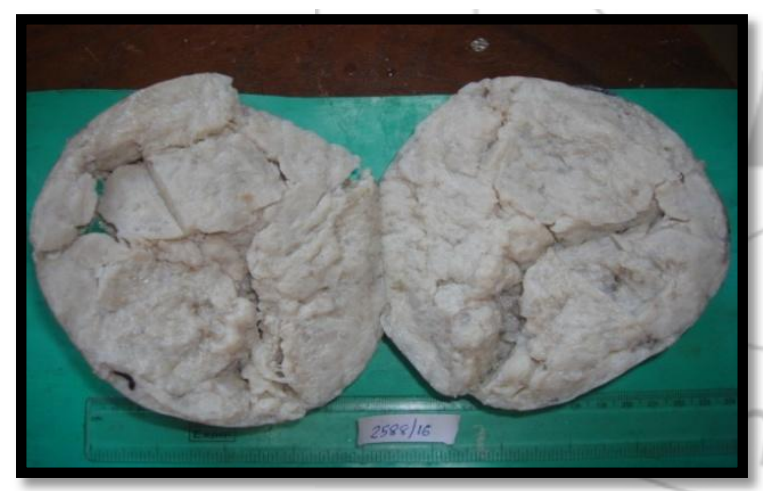

Figure 5: Cut section of the specimen

Histopathological examination (Fig.6) revealed paucicellular lesions with extensive areas of hyalinisation. Spindle cells were seen in fascicles and widely scattered by hyalinisation. Perivascular cellularity was more. Vague palisading was also seen. There were no atypia, mitosis or necrosis. Features were suggestive of leiomyoma.

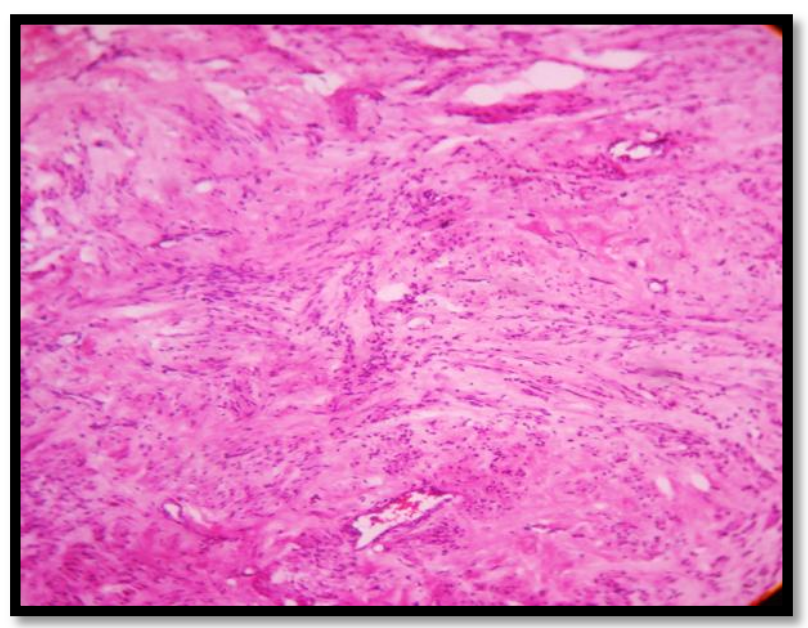

Figure 6: Microscopic picture of the specimen showing spindle cells in fascicles with scattered hyalinisation

\section{Discussion}

Leiomyomas are the commonest benign uterine tumours occurring in about $20 \%$ to $30 \%$ of premenopausal women ${ }^{(1)}$. The incidence reduces in postmenopausal women as it is believed that hormonal changes assist spontaneous resolution of such tumours. Extra uterine leiomyomas are rare, and occur due to seeding of myomatous tissues after gynecological surgeries. It is more common after laparoscopic gynecologic procedures $(2,3,4)$. In those receiving hormone replacement therapy, smooth muscle tumours may originate in the abdominal wall from cells of vessel wall. Leiomyoma originates from smooth muscle cells of the uterus and rarely from intestine or vessel wall. The alteration of such cells to leiomyoma involves somatic mutation and unknown synergistic action of hormones, deranged lipoid metabolism and local growth factors ${ }^{(5)}$. This transformation of smooth muscle cell may be due to progesterone and insulin like growth factors ${ }^{(5)}$. Although lipoleiomyoma arising from anterior abdominal wall without the presence of above factors have been reported before, isolated leiomyoma arising de novo in the anterior abdominal wall in pre-peritoneal space has not been reported so far.

A variety of mechanisms have been proposed for the initiation of tumorigenesis necessary for leiomyoma formation in uterus. Hypotheses include genetic predisposition, menstrual cycle-related mitotic dysregulation, myometrial cell phenotype transformation in response to ischemia, and an inherent myometrial predisposition to leiomyoma formation. Despite the abundance of theories and evidentiary support, a specific common cause is yet to be identified in leiomyoma formation.

Well differentiated tumours arising from fat cells, fibrous tissue, and muscle or mesothelial cells can be considered as the differential diagnosis of pre-peritoneal mass. Such tumours are usually benign. Malignant transformation occurs in disseminated variety ${ }^{(5,6)}$. The diagnosis of de novo leiomyoma of the anterior abdominal wall is made only when there is no history of previous abdominal surgery for leiomyoma of uterus. In cases where a leiomyoma has been 


\section{International Journal of Science and Research (IJSR) \\ ISSN (Online): 2319-7064 \\ Index Copernicus Value (2015): 78.96 | Impact Factor (2015): 6.391}

removed previously, seeding of the scar can give rise to tumour later ${ }^{(5,6)}$.

Parasitic leiomyomas also have been reported in the retro or pre-peritoneum. The uterine mass adheres to these structures and develops its own blood supply and loses its attachment over time from the organ of origin, and develops as a 'parasite' at a new location ${ }^{(5,6,7)}$. Our case is unusual in that there were no gynaecologic complaints or any history of gynaecologic surgery.

The tumour should be removed enbloc and morcellation avoided to prevent seeding/recurrence of the tumour. In case of large defect arising after tumour excision, synthetic mesh can be used for abdominal wall closure ${ }^{(6)}$.

\section{Conclusion}

A leiomyoma, although commonly seen in uterus or gastrointestinal tract, can develop de novo in areas devoid of myomatous tissues or smooth muscle cells. Whether smooth muscle cells in vessels of anterior abdominal wall react to extraneous hormonal stimulation to form a de novo leiomyoma is yet to be established. This condition has to be considered as a differential diagnosis of anterior abdominal wall tumours. Enbloc removal is the treatment of choice for this condition.

\section{References}

[1] Je Young Cho, Ji Young Woo, Hye-Suk Hong et al. Anterior Abdominal Wall Leiomyoma Arising De Novo in a Fertile Women: A Case Report. J Korean Soc Radiol 2016;74(1):71-74

[2] Manojit Midya, Neeraj K. Dewanda. Primary Anterior Abdominal Wall Leiomyoma- A Diagnostic Enigma. $J$ Clin Diagn Res. 2014 Oct; 8(10): NJ01-NJ02

[3] Abderlrahman et al., 2014, Solitary Abdominal Wall Leiomyoma: 2 Cases Reports with Immunohistochemical Stain Confirmation and Literature Review, International Journal of Clinical Case Reports, Vol.4 No.8 1-4

[4] Gabriel O Igberase, Tagbire O Mabiaku, Peter N Ebeigbe, Harrison $\mathrm{O}$ Abedi. Solitary anterior abdominal wall leiomyoma in a 31-year-old multipara woman: a case report. Cases J. 2009; 2: 113

[5] K.L. Narasimha Rao et al. Solitary Anterior Abdominal Wall Leiomyoma Arising from Tubectomy Scar. IOSR Journal of Dental and Medical Sciences Vol.13 Issue 1 Ver. 3 (Jan. 2014), PP 4-6

[6] Hamed A. Al-Wadaani. Anterior Abdominal Wall Leiomyoma Arising De Novo in a Perimenopausal Woman. Oman Med J.2012 Jul; 27(4): 323-325

[7] Narayanaswamy M, Bhaskaran A, Kalyani R, Yamini Agarwal. Anterior Abdominal Wall Leiomyoma Without Antecedent Pelvic Surgery Mimicking an Ovarian Tumour. J Clin Biomed Sci 2011 ; 1 (2) 